\title{
Dedifferentiation, Transdifferentiation, and Proliferation: Mechanisms Underlying Cardiac Muscle Regeneration in Zebrafish
}

\author{
Kazu Kikuchi
}

Published online: 30 January 2015

(c) The Author(s) 2015. This article is published with open access at Springerlink.com

\begin{abstract}
The adult mammalian heart is increasingly recognized as a regenerative organ with a measurable capacity to replenish cardiomyocytes throughout its lifetime, illuminating the possibility of stimulating endogenous regenerative capacity to treat heart diseases. Unlike mammals, certain vertebrates possess robust capacity for regenerating a damaged heart, providing a model to understand how regeneration could be augmented in injured human hearts. Facilitated by its rich history in the study of heart development, the teleost zebrafish Danio rerio has been established as a robust model to investigate the underlying mechanism of cardiac regeneration. This review discusses the current understanding of the endogenous mechanisms behind cardiac regeneration in zebrafish, with a particular focus on cardiomyocyte dedifferentiation, transdifferentiation, and proliferation.
\end{abstract}

Keywords Regeneration - Zebrafish · Cardiomyocyte · Dedifferentiation - Transdifferentiation · Proliferation

\section{Introduction}

Myocardial infarction is a leading cause of death worldwide. Urgently needed are the therapies that facilitate

This article is part of the Topical Collection on Development.

K. Kikuchi $(\bowtie)$

Developmental and Stem Cell Biology Division, Victor Chang Cardiac Research Institute, Darlinghurst, NSW 2010, Australia e-mail: k.kikuchi@victorchang.edu.au

K. Kikuchi

St. Vincent's Clinical School, University of New South Wales, Kensington, NSW 2052, Australia replacement of damaged cardiac tissue with new functional cardiomyocytes. In principle, the damaged heart could be regenerated by transplanting cardiomyocytes differentiated in vitro from a variety of cellular sources such as endogenous cardiac stem/progenitor cells [1,2], embryonic stem cells [3-5], and induced pluripotent stem cells [6]. Alternatively, cardiomyocytes could be directly generated from cardiac fibroblasts in vitro and in vivo by overexpressing defined cardiac transcription factors [7-11] or MicroRNAs (miRNAs) [12].

Another approach would be to identify successful examples of cardiac regeneration in nature, elucidate their underlying mechanisms, and then attempt to apply the insights gained to humans through the provision of the appropriate regenerative stimuli. Urodele amphibians and teleosts are well-known examples of animals that possess remarkable regenerative capacity in a variety of structures and organs as adults $[13,14]$. Among these, the zebrafish arguably displays the most robust and best characterized cardiac regenerative responses known till date [15-20]. This review will discuss the mechanism underlying cardiac regeneration in zebrafish with a particular focus on cardiomyocyte dedifferentiation, transdifferentiation, and proliferation.

\section{Evidence and Mechanisms Underlying Cardiomyocyte Dedifferentiation}

Genetic fate-mapping studies conducted by Poss and Izpisua Belmonte groups have provided convincing evidence that new myocardium is generated from differentiated cardiomyocytes during zebrafish heart regeneration [21••, 22••] (Fig. 1). In both studies, two transgenic zebrafish strains that were essentially the same were used: one line 
was an inducible Cre line in which the promoter of cardiac myosin light chain 2 ( $\mathrm{cmlc} 2 / \mathrm{myl}$ ) gene drives the myocardial expression of tamoxifen-inducible Cre recombinase (CreER) and the other is an indicator line in which enhanced green fluorescent protein (EGFP) will be expressed in $\mathrm{CreER}^{+}$cells, only when CreER excises a loxP-flanked stop cassette after tamoxifen treatments. In the double transgenic context of these lines, almost all $\mathrm{Cmlc}^{+}$cardiomyocytes were labeled with EGFP in the uninjured heart by tamoxifen treatments, enabling the examination of the extent of contribution of existing cardiomyocytes to the regenerated cardiac tissue. After the labeling, the hearts were injured at the ventricular apex with resection surgery, removing approximately $20 \%$ of the ventricular muscle, and histologically examined at 30 days post-injury, a timepoint at which regeneration is normally completed. The result clearly showed that the majority of the regenerated myocardium retains EGFP expression, with no significant difference detected in the proportion of the $\mathrm{EGFP}^{+}$muscle in the regenerated tissue compared with that in the uninjured tissue. Thus, existing $\mathrm{Cmlc2}^{+}$cardiomyocytes, but not $\mathrm{Cmlc}^{-}$non-myocytes, are the major source for new cardiac muscle during zebrafish heart regeneration (Fig. 1). The same result has been observed during the regeneration after genetic ablation of cardiomyocytes [20], in which nearly $60 \%$ of the entire muscle was depleted, as well as during the regeneration of the neonatal mouse heart [23••], suggesting that differentiated cardiomyocytes are the robust source for creating new muscle in natural cardiac regeneration models.

Regenerating cardiomyocytes in the zebrafish heart has unique morphological and molecular signatures distinct from that seen in non-regenerating cardiomyocytes. Histological analyses using transmission electron microscopy and immunofluorescence staining have revealed that cardiomyocytes at the injury border zone acquire lessorganized sarcomeres with reduced Z-bands, undergoing DNA synthesis and mitosis $[21 \bullet \bullet, 22 \bullet \cdot]$. Analyses of fluorescence reporter strains have shown that the regulatory sequences of cardiogenic transcription factors such as

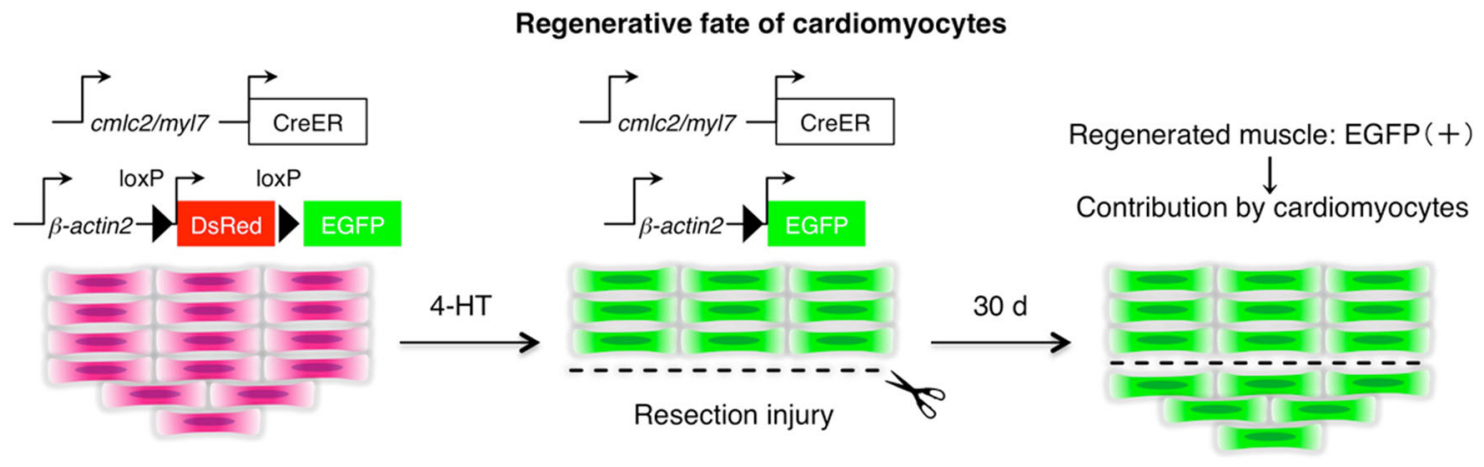

Molecular mechanisms regulating cardiomyocyte proliferation

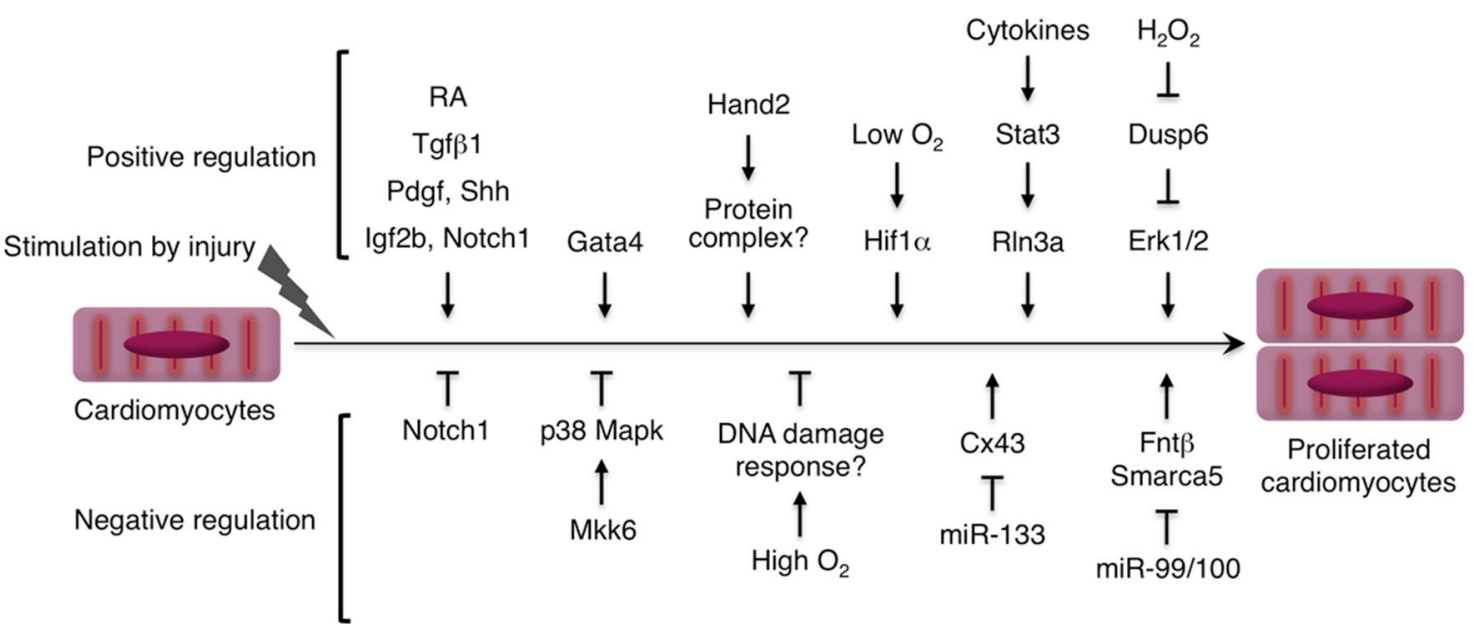

Fig. 1 Genetic fate-mapping experiments have provided conclusive evidence that spared cardiomyocytes $\left(\mathrm{EGFP}^{+}\right)$, but not non-myocytes $\left(\mathrm{EGFP}^{-}\right)$, are the major source for new cardiac muscle regenerated at the wound area. After injury, cardiomyocytes are activated and proliferate to regenerate lost myocardium, which is positively and negatively regulated by various intrinsic and extrinsic mechanisms. 4-HT 4-hydroxytamoxifen 
Gata 4 and Hand 2 are activated in cardiomyocytes adjacent to the wound area, and such profiles are maintained throughout the regeneration process [21••, 24]. Together with the observation that the expression of other embryonic cardiogenesis genes is also induced in the injured heart [25, 26], these data suggest that zebrafish cardiomyocytes reduce their contractile state upon injury, acquiring immature phenotypes that likely facilitate cell division. Interestingly, the morphological and molecular features described above are reminiscent of those observed in mammalian cardiomyocytes during development. During the development of the mouse heart, disassembly of sarcomere structures have been detected in proliferating cardiomyocytes [27], and the Gata4 gene is expressed in the myocardium and is functionally required for cardiomyocyte proliferation [28], suggesting that mechanisms underlying cardiomyocyte dedifferentiation and proliferation are conserved between zebrafish and mammals.

The notion that molecular programs of zebrafish cardiomyocyte dedifferentiation and proliferation might be conserved in the non-regenerative mammalian heart has been addressed in a recently published study [29]. To identify a conserved mechanism, Aguirre et al. focused on miRNAs, small non-coding RNA molecules that regulate gene expression at post-transcriptional levels, given their wide potential to regulate gene expression changes. They identified that two miRNA families, miR-99/100 and let$7 \mathrm{a} / \mathrm{c}$, are sharply downregulated during zebrafish heart regeneration, and they provided evidence that miR-99/100 negatively regulates cardiomyocyte proliferation and regeneration. miR-99/100 targets two proteins: (1) Fnt $\beta$, the beta subunit of farnesyl-transferase and (2) Smarca5, a SWI/SNF family chromatin regulator; a pharmacological blockade of Fnt $\beta$ reduced cardiomyocyte proliferation and impaired regeneration. Unlike the zebrafish heart, the expression of miR-99/100 was unchanged in the mouse heart before and after injury; however, when miR-99/100 was forcefully downregulated in cultured cells, adult mouse cardiomyocytes disassembled sarcomere, reexpressed GATA4, and induced proliferation. These phenotypes were not observed when both miR-99/100 and its protein targets were depleted, indicating that FNT $\beta$ and SMARCA5 play a major role in the regulation controlled by the miR-99/100 pathway. Finally, the authors examined the role of the miR-99/100 pathway in the infarcted mouse heart and showed that miR-99/100 and let-7a/c knockdown significantly increases the number of cardiomyocytes with proliferation and dedifferentiation phenotypes, reducing scar size and improving cardiac function. These results indicated that the miR-99/100 pathway is dormant and yet can be reactivated to induce cardiomyocyte dedifferentiation and proliferation in mammalian hearts [29]. It remains unclear how FNT $\beta$ and SMARCA5 promote regeneration; however, the study described above supports a concept that deciphering molecular mechanisms underlying cardiac regeneration in zebrafish will provide insights into how endogenous regeneration can be enhanced in mammalian hearts.

\section{Transdifferentiation and Cardiac Muscle Regeneration}

Transdifferentiation is a regenerative phenomenon in which one cell type transforms to another, occasionally via an undifferentiated intermediate, and has been reported as a mechanism observed during natural regeneration in certain vertebrates. A classic example is lens regeneration in adult newts, in which a new lens is formed from the dorsal pigmented iris [30]. Transdifferentiation may also play a critical role in limb regeneration in urodele amphibians. During axolotl limb regeneration, although most cell types maintain their lineages within the blastema, dermis-derived cells re-differentiate into cartilage and tendons likely through transdifferentiation [31]. Little is known about how transdifferentiation is naturally induced upon injury, but a key event during this remarkable process would be the change in the epigenetic state of differentiated cells.

Given our understanding of the epigenetic stability of differentiated states, it seems that such a change would only occur in developmentally related lineages, and therefore, transdifferentiation may not be commonly observed in other regeneration models under natural conditions. However, it is increasingly recognized that epigenetic states can be reprogrammed by manipulating gene regulatory networks, which is most notably shown by the induction of pluripotent stem cells from adult somatic cells by the expression of defined transcription factors [32]. More recent studies have demonstrated that the overexpression of cardiac transcription factors [7-11] or miRNAs [12] can convert non-cardiomyocytes into functional cardiomyocytes.

Transdifferentiation may occur naturally to accommodate extreme loss of a particular cell lineage. When nearly all pancreatic $\beta$ cells are depleted in the adult mouse pancreas, glucagon-producing $\alpha$ cells, which would otherwise never change its lineage, are shown to transdifferentiate into insulin-producing $\beta$-like cells, reverting hyperglycemia to normoglycemia [33]. Chi and colleagues have recently revealed that a similar mechanism contributes to cardiac muscle regeneration in the zebrafish heart [34•]. The authors generated a transgenic line in which ventricular cardiomyocytes can be depleted in a temporally regulated manner and found that zebrafish embryos completely regenerate ventricular myocardium within 4 days after massive ablation of ventricular muscle. To address the mechanism underlying this remarkable regeneration, the 
authors performed immunofluorescence staining and found that mitotic response is elevated not only in the regenerating ventricle but also in the atrium. Live-imaging and genetic fate-mapping experiments revealed that after the massive ablation of the ventricular muscle, atrial cardiomyocytes migrate into the damaged ventricle, expanding across the chamber, and regenerate ventricular myocardium.

To understand the molecular mechanism underlying the atrial-to-ventricular transdifferentiation, the authors performed gene expression analyses and found that the ventricular muscle depletion activates the re-expression of various heart field marker genes and the ectopic expression of Notch pathway genes in the damaged heart. Pharmacological inhibition of the Notch pathway impaired ventricular muscle regeneration after the ablation. Interestingly, using a reporter zebrafish strain in which cells receiving Notch signaling can be visualized by EGFP expression, the authors observed the activation of Notch signaling in the atrial endocardium, but not in the myocardium during regeneration, indicating that atrial-to-ventricular transdifferentiation is indirectly regulated by Notch signaling through the activation of the atrial endocardium $[34 \bullet]$.

The results described above were obtained from zebrafish embryos, and the atrial-to-ventricular transdifferentiation was not observed in the adult heart [34•], which suggests that the transdifferentiation capacity differs depending on age. Interestingly, a recent study using the aforementioned $\beta$ cell ablation model has shown that somatostatin-producing $\delta$ cells, not the glucagon-producing $\alpha$ cells, transdifferentiate into $\beta$-like cells in 2 -week-old mice after massive ablation of $\beta$ cells [35]. Similarly, transdifferentiation to ventricular muscle might occur in the adult heart more prominently from a cell type different from atrial cardiomyocytes. The atrial-to-ventricular transdifferentiation described above depends on the endocardial activation by the Notch pathway [34•]. This mechanism seems reminiscent of the role of the endocardium in the regenerating adult heart, in which endocardial cells produce various paracrine factors, promoting the local proliferation of cardiomyocytes at the regenerating area [26]. Interesting future experiments would comprise the investigation of the factors that are released from the endocardium after Notch activation and identification of a direct mechanism to induce genetic programs for atrial-toventricular transdifferentiation.

\section{Molecular Regulation of Cardiomyocyte Proliferation}

Given the identification of existing cardiomyocytes as the dominant source for the regenerated myocardium in the zebrafish heart, the mechanism underlying cardiomyocyte proliferation is increasingly recognized as a major area of interest within the field (Fig. 1). Thus far, a number of paracrine factors have been identified to be released in the regenerating area from the epicardium, the outermost mesothelial tissue, and/or from the endocardium, the innermost endothelial tissue. These factors include retinoic acid (RA) and developmental growth factors such as Tgf $\beta 1$, Pdgf, Shh, and Igf $2 b$; the expression of these genes are maintained throughout the regeneration process near the wound area and promote local proliferation of cardiomyocytes [25, 26, 36-39]. The expression of the Notch family genes is also induced at the injury site [40, 41]; interestingly, either inhibiting or activating the Notch pathway similarly blocked cardiomyocyte proliferation and regeneration, suggesting that successful regeneration of the zebrafish heart requires a fine balance between the activation and inhibition of the Notch pathway [41].

As mentioned earlier, the expression of the cardiogenic transcription factor genes is upregulated in regenerating cardiomyocytes $[21 \bullet \cdot, 24-26]$. Among these, the function of Gata 4 and Hand2 has recently been examined during cardiac regeneration. Gupta et al. overexpressed a dominant-negative form of Gata4 (g4DN) in cardiomyocytes and examined the effect of Gata4 inhibition during cardiac growth and regeneration [42]. When $g 4 D N$ expression was induced in juvenile zebrafish, multiple signs of heart failure, including lethargy, gasping behavior, as well as edema were observed, and the animal survival was sharply reduced to approximately $16 \%$ of the control level; when induced in adult zebrafish, such phenotypes were not observed, suggesting that Gata4 has an essential role for maintaining cardiac function during growth. The authors next performed regeneration experiments after myocardial induction of $g 4 D N$ and found that with $g 4 D N$ expression, myocardial proliferation is reduced and heart regeneration is arrested with collagenous scar formation at the wound area. Interestingly, although $g 4 D N$ was induced in the entire myocardium, the proliferation of cardiomyocytes was normal in the inner trabecular muscle but severely blocked in the lateral ventricular wall muscle [42], where the activation of the gata4 promoter has been detected [21••], which suggests that the proliferation of trabecular cardiomyocytes is regulated by a Gata4-independent mechanism.

More recently, Yelon and colleagues generated a new transgenic strain that allows tamoxifen-inducible hand2 expression, in combination with the myocardial specific CreER strain, and examined whether Hand 2 overexpression affects the regenerative capacity of cardiomyocytes [24]. When hand 2 expression was induced in the injured heart, no effect was observed in myocardial proliferation; however, when induced after injury, proliferation was 
significantly elevated in cardiomyocytes in the vicinity of the wound area, indicating that hand 2 overexpression promotes cardiomyocyte production during regeneration. The authors also confirmed that the hand 2 overexpression increases the number of cardiomyocytes in the early zebrafish embryos. Interestingly, this increase was similarly induced by the expression of a Hand 2 mutant lacking the DNA-binding domain but not by the one lacking the dimerization domain, suggesting that Hand 2 regulates the proliferation of cardiomyocytes by interacting as multimeric complexes [24].

Both Gata4 and Hand2 have been known to have a capacity for converting mammalian cardiac fibroblasts into cardiomyocyte-like cells, when overexpressed with other cardiac transcription factors such as Mef2c and Tbx5 [711]. Given that all these factors are upregulated in the regenerating area of the zebrafish heart, it would be interesting to speculate that the injury-induced upregulation of cardiogenic transcription factors might have an instructive role in inducing immature phenotypes in cardiomyocytes during cardiac regeneration.

Regeneration is a phenomenon initiated by injury, and therefore, it appears logical to speculate that key signals to initiate regenerative responses may be provided by immediate events provoked by injury. Given the high oxygen demand in contracting myocardium, tissue hypoxia is a reasonable candidate for an immediate event after injury. Jopling et al. have recently addressed how hypoxia affects heart regeneration in zebrafish [43•]. The authors first performed regeneration experiments under severe anemia, induced by treating fish with a hemolytic chemical reagent, and found that cardiomyocyte proliferation is elevated in anemic zebrafish during regeneration. They next generated a transgenic strain that enables Cre-dependent expression of the dominant-negative form of hypoxia-inducible factor $\alpha(\mathrm{dnHIF} \alpha)$, a transcription factor essential for the cellular response to hypoxia, and performed regeneration experiments using the double transgenic fish crossed with a cardiomyocyte-specific CreER strain. With dnHIF $\alpha$ expression, DNA synthesis was reduced in cardiomyocytes and cardiac muscle regeneration appeared to be incomplete at 1 month after the injury, indicating that Hif $\alpha$-mediated hypoxia signaling is essential for cardiac muscle regeneration in zebrafish. When injured zebrafish were maintained in a hyperoxic condition during the period of regeneration, cardiomyocyte proliferation was decreased and cardiac muscle regeneration was impaired. Together, these results indicated that environmental oxygen concentration regulates cardiomyocyte proliferation in the regenerating zebrafish heart [43•], which is consistent with a recent finding that a higher oxygen concentration in the post-natal environment of the mouse heart limits the proliferation of cardiomyocytes [44•].
Acute inflammatory response is another immediate event induced by injury. Although inflammation appears to be intimately associated with fibrotic responses and regeneration failure observed in most adult mammalian tissues [45], a recent study has demonstrated that acute inflammatory signals can promote regeneration of damaged nervous tissues in adult zebrafish [46]. Yi et al. have recently performed cardiomyocyte-specific unbiased gene expression analyses using regenerating zebrafish heart samples and found that the most prominently upregulated are the genes for inflammatory cytokines and for Jak/Stat3 pathway members, molecules critical for transducing inflammatory signals [47]. The authors generated a transgenic line that enables inducible expression of dominantnegative Stat3 (dnStat3) after the excision of loxP-flanked stop sequences, and crossed this line with the $\mathrm{cmlc2}$ : CreER line to perform cardiomyocyte-specific loss-of-function analysis of the Stat3 pathway. Regeneration tests using the double transgenic strain revealed that Stat 3 inhibition reduced cardiomyocyte proliferation to nearly $20 \%$ of the control level and blocked myocardial regeneration with the formation of a collagenous scar at the wound area. By comparing gene expression profiles obtained from ventricles with or without dnStat3, the authors identified Relaxin 3a $(\mathrm{R} \ln 3 \mathrm{a})$, a ligand for a $\mathrm{G}$ protein-coupled receptor [48], as a candidate downstream molecule of Stat 3 in the regenerating heart. Chromatin immunoprecipitation revealed that Stat3 was recruited to the rln3a promoter region upon cardiac injury, and injection of recombinant human RLN3 partially rescued the impaired proliferation of cardiomyocytes with dnStat3. Thus, during zebrafish heart regeneration, inflammatory cytokines induced by injury likely lead to Stat 3 activation in cardiomyocytes, promoting the myocardial release of $\mathrm{R} \ln 3 \mathrm{a}$, in turn inducing regenerative proliferation of cardiomyocytes [47].

The reactive oxygen species hydrogen peroxide $\left(\mathrm{H}_{2} \mathrm{O}_{2}\right)$ has been identified as one of the earliest signals released from damaged tissues to induce inflammatory responses [49]. Xiong and colleagues have recently established a transgenic reporter line with which the $\mathrm{H}_{2} \mathrm{O}_{2}$ level can be monitored and found that $\mathrm{H}_{2} \mathrm{O}_{2}$ production is markedly upregulated in the epicardium near the wound area [50]. Pharmacological inhibition of $\mathrm{H}_{2} \mathrm{O}_{2}$ production as well as transgenic overexpression of catalase, a scavenger enzyme for $\mathrm{H}_{2} \mathrm{O}_{2}$, impaired cardiac muscle regeneration with collagenous scar formation, suggesting that the injury-induced $\mathrm{H}_{2} \mathrm{O}_{2}$ signal is required for regenerative proliferation of cardiomyocytes. Biochemical analyses using cell lines and zebrafish embryos revealed that elevated $\mathrm{H}_{2} \mathrm{O}_{2}$ promotes ubiquitination-dependent protein degradation of Dusp6, a redox-sensitive phosphatase that negatively regulates MAP kinase signaling. Together, these results suggested that $\mathrm{H}_{2} \mathrm{O}_{2}$ plays a critical role in priming cardiomyocyte 
proliferation after injury by unlocking the suppression of MAP kinase mediated by Dusp6 [50].

Regenerative proliferation needs to be downregulated as the damaged tissue is restored. Although knowledge remains limited, several studies have reported mechanisms underlying this regulation as described below. Consistent with the results obtained from mouse studies, a recent zebrafish study showed that phosphorylated-p38 Mapk is present in the nuclei of non-proliferating cardiomyocytes but disappears when cardiomyocytes enter the mitotic cycle in vitro and in vivo [51]. The myocardial overexpression of the constitutive active form of Mkk6, an upstream kinase of p38 Mapk, was shown to block cardiomyocyte proliferation and arrest cardiogenesis at the wound area.

A mechanism underlying negative regulation of cardiomyocyte proliferation has also been attributed to miRNAs. As described earlier, miR-99/100 represses regenerative proliferation of cardiomyocytes by downregulating the expression of Fnt $\beta$ and Smarca5 [29]. Unbiased miRNA expression analyses on regenerating hearts have also revealed that the expression of miR-133, a mammalian ortholog of which contributes to cardiac development and diseases [52, 53], inversely correlates with the progress of cardiac regeneration in zebrafish. Constitutive expression of miR-133 was detected in cardiomyocytes in the uninjured heart, and its overexpression was shown to reduce cardiomyocyte proliferation by nearly $50 \%$ and block myocardial regeneration with collagenous scar formation at the wound area. By contrast, the proliferation cardiomyocytes was elevated when miR133 molecules were removed by expressing "sponge" sequences, which contain triplicates of perfect-match binding sites for miR-133. As a novel target of miR-133, the authors identified connexin 43 (Cx43), which is not only widely known as a gap junction protein but has also been reported to regulate cell proliferation and growth, and showed that the pharmacological inhibition of cx43 function blocks regenerative proliferation of cardiomyocytes. Together, these results indicated that miR-133 negatively regulates cardiomyocyte proliferation, partly by modulating the Cx43 level, during cardiac regeneration in zebrafish [54].

\section{Conclusion}

Zebrafish hearts regenerate mainly through cardiomyocyte dedifferentiation and proliferation [21••, 22••], a mechanism also underlying the regeneration of the neonatal mouse heart [23••]. The regenerative capacity of the neonatal mouse heart diminishes within a week, seemingly concomitant with cardiomyocyte binucleation [55]; however, even after this period, robust proliferation has recently been shown to occur in binucleated cardiomyocytes at post-natal day 15 , establishing the final number of cardiomyocytes in the adult heart [56••]. Cardiomyocytes in the adult mammalian heart do not appear to have a significant capacity for proliferation in the injury setting, but they maintain a measurable capacity for

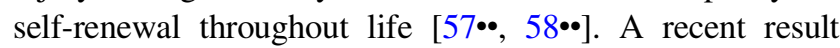
supports the notion that mechanisms underlying regeneration of the zebrafish heart are conserved in mammalian hearts and can be reactivated to induce regeneration [29]. Further understanding of the cellular and molecular regulation of cardiac regeneration in zebrafish will provide valuable insights for a better understanding of how the human heart can be stimulated to reactivate its endogenous regenerative capacity as a novel therapeutic strategy to treat cardiac diseases.

Acknowledgments I apologize to my colleagues whose work was not discussed due to space limitations. K. K. is an Australian Research Council Future Fellow (FT110100836) and acknowledges the grant support from the National Health and Medical Research Council (1032522 and 1046469).

Open Access This article is distributed under the terms of the Creative Commons Attribution License which permits any use, distribution, and reproduction in any medium, provided the original author(s) and the source are credited.

\section{Compliance with Ethics Guidelines}

Conflict of Interest Kazu Kikuchi declares no conflict of interest.

Human and Animal Rights and Informed Consent This article does not contain any studies with human or animal subjects performed by any of the authors.

\section{References}

Papers of particular interest, published recently, have been highlighted as:

- Of importance

-• Of major importance

1. Bu L, Jiang X, Martin-Puig S et al (2009) Human ISL1 heart progenitors generate diverse multipotent cardiovascular cell lineages. Nature 460:113-117

2. Bearzi C, Rota M, Hosoda T et al (2007) Human cardiac stem cells. Proc Natl Acad Sci USA 104:14068-14073

3. Chong JJH, Yang X, Don CW et al (2014) Human embryonicstem-cell-derived cardiomyocytes regenerate non-human primate hearts. Nature 510:273-277

4. Laflamme MA, Chen KY, Naumova AV et al (2007) Cardiomyocytes derived from human embryonic stem cells in pro-survival factors enhance function of infarcted rat hearts. Nat Biotechnol 25:1015-1024 
5. Mummery C, Ward-van Oostwaard D, Doevendans P et al (2003) Differentiation of human embryonic stem cells to cardiomyocytes: role of coculture with visceral endoderm-like cells. Circulation 107:2733-2740

6. Zhang J, Wilson GF, Soerens AG et al (2009) Functional cardiomyocytes derived from human induced pluripotent stem cells. Circ Res 104:e30-e41

7. Nam Y-J, Song K, Luo X et al (2013) Reprogramming of human fibroblasts toward a cardiac fate. Proc Natl Acad Sci USA 110:5588-5593

8. Qian L, Huang Y, Spencer CI et al (2012) In vivo reprogramming of murine cardiac fibroblasts into induced cardiomyocytes. Nature 485:593-598

9. Song K, Nam Y-J, Luo X et al (2012) Heart repair by reprogramming non-myocytes with cardiac transcription factors. Nature 485:599-604

10. Ieda M, Fu J-D, Delgado-Olguin P et al (2010) Direct reprogramming of fibroblasts into functional cardiomyocytes by defined factors. Cell 142:375-386

11. Nam Y-J, Lubczyk C, Bhakta M et al (2014) Induction of diverse cardiac cell types by reprogramming fibroblasts with cardiac transcription factors. Development 141:4267-4278

12. Jayawardena TM, Egemnazarov B, Finch EA et al (2012) MicroRNA-mediated in vitro and in vivo direct reprogramming of cardiac fibroblasts to cardiomyocytes. Circ Res 110:1465-1473

13. Brockes JP, Kumar A (2008) Comparative aspects of animal regeneration. Annu Rev Cell Dev Biol 24:525-549

14. Poss KD (2010) Advances in understanding tissue regenerative capacity and mechanisms in animals. Nat Rev Genet 11:710-722

15. Chablais F, Veit J, Rainer G, Jaźwińska A (2011) The zebrafish heart regenerates after cryoinjury-induced myocardial infarction. BMC Dev Biol 11:21

16. González-Rosa JM, Martín V, Peralta M et al (2011) Extensive scar formation and regression during heart regeneration after cryoinjury in zebrafish. Development 138:1663-1674

17. Parente V, Balasso S, Pompilio G et al (2013) Hypoxia/reoxygenation cardiac injury and regeneration in zebrafish adult heart. PLoS One 8:e53748

18. Poss KD, Wilson LG, Keating MT (2002) Heart regeneration in zebrafish. Science 298:2188-2190

19. Schnabel K, Wu C-C, Kurth T, Weidinger G (2011) Regeneration of cryoinjury induced necrotic heart lesions in zebrafish is associated with epicardial activation and cardiomyocyte proliferation. PLoS One 6:e18503

20. Wang J, Panáková D, Kikuchi K et al (2011) The regenerative capacity of zebrafish reverses cardiac failure caused by genetic cardiomyocyte depletion. Development 138:3421-3430

21. • Kikuchi K, Holdway JE, Werdich AA et al (2010) Primary contribution to zebrafish heart regeneration by gata4+ cardiomyocytes. Nature 464:601-605. This paper provide evidence that cardiomyocytes are the major cellular source for new cardiac muscle in natural regeneration models

22. • Jopling C, Sleep E, Raya M et al (2010) Zebrafish heart regeneration occurs by cardiomyocyte dedifferentiation and proliferation. Nature 464:606-609. This paper provide evidence that cardiomyocytes are the major cellular source for new cardiac muscle in natural regeneration models

23. • Porrello ER, Mahmoud AI, Simpson E et al (2011) Transient regenerative potential of the neonatal mouse heart. Science 331:1078-1080. This paper provide evidence that cardiomyocytes are the major cellular source for new cardiac muscle in natural regeneration models

24. Schindler YL, Garske KM, Wang J et al (2014) Hand2 elevates cardiomyocyte production during zebrafish heart development and regeneration. Development 141:3112-3122
25. Lepilina A, Coon AN, Kikuchi K et al (2006) A dynamic epicardial injury response supports progenitor cell activity during zebrafish heart regeneration. Cell 127:607-619

26. Kikuchi K, Holdway JE, Major RJ et al (2011) Retinoic acid production by endocardium and epicardium is an injury response essential for zebrafish heart regeneration. Dev Cell 20:397-404

27. Ahuja P, Perriard E, Perriard J-C, Ehler E (2004) Sequential myofibrillar breakdown accompanies mitotic division of mammalian cardiomyocytes. J Cell Sci 117:3295-3306

28. Zeisberg EM, Ma Q, Juraszek AL et al (2005) Morphogenesis of the right ventricle requires myocardial expression of Gata4. J Clin Invest 115:1522-1531

29. Aguirre A, Montserrat N, Zachiggna S, Nivet E (2014) In vivo activation of a conserved microRNA program induces mammalian heart regeneration. Cell Stem Cell. doi: 10.1016/j.stem.2014. 10.003

30. Eguchi G, Abe SI, Watanabe K (1974) Differentiation of lens-like structures from newt iris epithelial cells in vitro. Proc Natl Acad Sci USA 71:5052-5056

31. Kragl M, Knapp D, Nacu E et al (2009) Cells keep a memory of their tissue origin during axolotl limb regeneration. Nature 460:60-65

32. Takahashi K, Yamanaka S (2006) Induction of pluripotent stem cells from mouse embryonic and adult fibroblast cultures by defined factors. Cell 126:663-676

33. Thorel F, Népote V, Avril I et al (2010) Conversion of adult pancreatic $\alpha$-cells to $\beta$-cells after extreme beta-cell loss. Nature 464:1149-1154

34. - Zhang R, Han P, Yang H et al (2013) In vivo cardiac reprogramming contributes to zebrafish heart regeneration. Nature 498:497-501. This study describes atrial-to-ventricular transdifferentiation after massive ablation of ventricular muscle in zebrafish embryos

35. Chera S, Baronnier D, Ghila L et al (2014) Diabetes recovery by age-dependent conversion of pancreatic $\delta$-cells into insulin producers. Nature 514:503-507

36. Chablais F, Jaźwińska A (2012) The regenerative capacity of the zebrafish heart is dependent on TGF $\beta$ signaling. Development 139:1921-1930

37. Huang Y, Harrison MR, Osorio A et al (2013) Igf signaling is required for cardiomyocyte proliferation during zebrafish heart development and regeneration. PLoS One 8:e67266

38. Kim J, Wu Q, Zhang Y et al (2010) PDGF signaling is required for epicardial function and blood vessel formation in regenerating zebrafish hearts. Proc Natl Acad Sci USA 107:17206-17210

39. Choi W-Y, Gemberling M, Wang J et al (2013) In vivo monitoring of cardiomyocyte proliferation to identify chemical modifiers of heart regeneration. Development 140:660-666

40. Raya A, Koth CM, Büscher D et al (2003) Activation of Notch signaling pathway precedes heart regeneration in zebrafish. Proc Natl Acad Sci USA 100(Suppl 1):11889-11895

41. Zhao L, Borikova AL, Ben-Yair R et al (2014) Notch signaling regulates cardiomyocyte proliferation during zebrafish heart regeneration. Proc Natl Acad Sci USA 111:1403-1408

42. Gupta V, Gemberling M, Karra R et al (2013) An injuryresponsive gata4 program shapes the zebrafish cardiac ventricle. Curr Biol 23:1221-1227

43. • Jopling C, Suñé G, Faucherre A et al (2012) Hypoxia induces myocardial regeneration in zebrafish. Circulation 126:3017-3027. This paper describe that a low $\mathrm{O}_{2}$ concentration promotes cardiomyocyte proliferation, whereas a high $\mathrm{O}_{2}$ concentration blocks it

44. - Puente BN, Kimura W, Muralidhar SA, et al. (2014) The oxygen-rich postnatal environment induces cardiomyocyte cellcycle arrest through DNA damage response. Cell 157:565-579. This paper describe that a low $\mathrm{O}_{2}$ concentration promotes 
cardiomyocyte proliferation, whereas a high $\mathrm{O}_{2}$ concentration blocks it

45. Forbes SJ, Rosenthal N (2014) Preparing the ground for tissue regeneration: from mechanism to therapy. Nat Med 20:857-869

46. Kyritsis N, Kizil C, Zocher S et al (2012) Acute inflammation initiates the regenerative response in the adult zebrafish brain. Science 338:1353-1356

47. Fang Y, Gupta V, Karra R et al (2013) Translational profiling of cardiomyocytes identifies an early Jak1/Stat 3 injury response required for zebrafish heart regeneration. Proc Natl Acad Sci USA 110:13416-13421

48. Hsu SY, Nakabayashi K, Nishi S et al (2002) Activation of orphan receptors by the hormone relaxin. Science 295:671-674

49. Niethammer P, Grabher C, Look AT, Mitchison TJ (2009) A tissue-scale gradient of hydrogen peroxide mediates rapid wound detection in zebrafish. Nature 459:996-999

50. Han P, Zhou X-H, Chang N et al (2014) Hydrogen peroxide primes heart regeneration with a derepression mechanism. Cell Res 24:1091-1107

51. Jopling C, Suñé G, Morera C, Izpisúa Belmonte JC (2012) p38a MAPK regulates myocardial regeneration in zebrafish. Cell Cycle 11:1195-1201

52. Liu N, Bezprozvannaya S, Williams AH et al (2008) microRNA133a regulates cardiomyocyte proliferation and suppresses smooth muscle gene expression in the heart. Genes Dev 22:3242-3254
53. Wystub K, Besser J, Bachmann A et al (2013) miR-1/133a clusters cooperatively specify the cardiomyogenic lineage by adjustment of myocardin levels during embryonic heart development. PLoS Genet 9:e1003793

54. Yin VP, Lepilina A, Smith A, Poss KD (2012) Regulation of zebrafish heart regeneration by miR-133. Dev Biol 365:319-327

55. Laflamme MA, Murry CE (2011) Heart regeneration. Nature 473:326-335

56. •• Naqvi N, Li M, Calvert JW et al (2014) A proliferative burst during preadolescence establishes the final cardiomyocyte number. Cell 157:795-807. This paper describes a novel finding that both mononucleated and binucleated cardiomyocytes proliferate and divide even after the neonatal period

57. •• Bergmann O, Bhardwaj RD, Bernard S et al (2009) Evidence for cardiomyocyte renewal in humans. Science 324:98-102. doi: 10.1126/science.1164680. This paper provide evidence that cardiomyocytes are replenished at a measureable rate in the adult mammalian heart

58. •- Senyo SE, Steinhauser ML, Pizzimenti CL, et al. (2013) Mammalian heart renewal by pre-existing cardiomyocytes. Nature 493:433-436. This paper provide evidence that cardiomyocytes are replenished at a measureable rate in the adult mammalian heart 